\title{
Circulating and peritoneal fluid interleukin-6 levels and gene expression in pelvic endometriosis
}

\author{
VÂNIA TEIXEIRA DE ANDRADE ${ }^{1 *}$, ANDRÉA PRESTES NÁCUL $^{2 *}$, BETÂNIA RODRIGUES DOS SANTOS $^{1}$, \\ SHEILA BÜNECKER LECKE ${ }^{1,3}$, POLI MARA SPRITZER ${ }^{1,4}$ and DÉBORA MARTINHO MORSCH ${ }^{1}$
}

\author{
${ }^{1}$ Gynecological Endocrinology Unit, Division of Endocrinology, Hospital de Clínicas de Porto Alegre, Porto Alegre, \\ Rio Grande do Sul 90035-003; ${ }^{2}$ Human Reproduction Unit, Hospital Fêmina, Grupo Hospitalar Conceição, Porto Alegre, \\ Rio Grande do Sul 90430-001; ${ }^{3}$ Department of Diagnostic Methods, Federal University of Health Sciences of Porto Alegre, \\ Porto Alegre, Rio Grande do Sul 90050-170; ${ }^{4}$ Laboratory of Molecular Endocrinology, Department of Physiology, \\ Federal University of Health Sciences of Porto Alegre, Porto Alegre, Rio Grande do Sul 90035-003, Brazil
}

Received February 4, 2016; Accepted October 4, 2016

DOI: 10.3892/etm.2017.4794

\begin{abstract}
Current data are inconsistent regarding the association between interleukin-6 (IL-6), a marker of acute phase inflammation, and pelvic endometriosis. The aim of the present study was to assess IL-6 levels in serum and peritoneal fluid (PF), as well as IL-6 gene expression in adipose tissue (AT) and endometrial samples in pelvic endometriosis. A total of 30 patients with endometriosis and 18 women with a normal pelvis were enrolled in this case-control study. IL-6 levels in PF and serum were determined using a human enzyme-linked immunosorbent assay and IL-6 gene expression was evaluated using reverse transcription-quantitative polymerase chain reaction. It was observed that IL-6 levels in the $\mathrm{PF}$ were higher in patients with endometriosis than in the control group $(\mathrm{P}=0.047)$ and patients with stage III/IV endometriosis exhibited higher IL-6 levels in the PF than those with stage I/II endometriosis and the control group $(\mathrm{P}<0.001)$. Furthermore, a strong correlation between PF IL-6 levels and the revised American Society for Reproductive Medicine score for endometriosis severity was identified $(r=0.77$; $\mathrm{P}<0.001)$. IL-6 gene expression did not differ significantly between endometriosis and control groups in endometrial samples or in AT of both groups. The results of the current study suggest that there may be an association between IL-6 and the presence and severity of pelvic endometriosis. The
\end{abstract}

Correspondence to: Dr Débora Martinho Morsch, Gynecological Endocrinology Unit, Division of Endocrinology, Hospital de Clínicas de Porto Alegre, 2350 Rua Ramiro Barcelos, Porto Alegre, Rio Grande do Sul 90035-003, Brazil

E-mail:dmmorsch@yahoo.com.br

*Contributed equally

Key words: endometriosis, adipose tissue, gene expression, interleukin-6, peritoneal fluid source of this higher IL-6 seems not to be specifically related to regional AT.

\section{Introduction}

Endometriosis is a chronic inflammatory condition characterized by implantation and growth of endometrial tissue outside the uterine cavity, which appears to depend on an abnormal peritoneal microenvironment consisting of increased levels of pro-inflammatory cytokines and growth factors (1).

Interleukin-6 (IL-6) is a glycoprotein secreted by a number of cell types including peritoneal macrophages, endometrial stromal cells and adipose tissue (AT) (2). AT has an abundant supply of inflammatory cytokines and contributes to $\sim 35 \%$ of circulating IL-6 $(1,3)$.

IL-6 is a marker of acute phase inflammation and performs a number of biological activities, such as the inducing the expression of vascular endothelial growth factor (4) and the growth and differentiation of B lymphocytes, as well as activating T lymphocytes (5). Additionally, IL-6 serves an important role in reproductive physiology, as it regulates folliculogenesis, ovarian steroid production and various early events associated with implantation (6). Previous studies have identified elevated IL- 6 concentrations in the peritoneal fluid (PF) of patients with endometriosis in comparison with controls without the disease (7-9), whereas others have failed to detect any differences $(10,11)$.

Different serum markers have been established that may identify patients in the initial stages of endometriosis, when ablation or excision of lesions improves reproductive outcomes (11-18). Among these markers, IL-6 appears to present good discriminatory power $(11,14,17)$. In turn, other studies have demonstrated that combined analyses of different serum markers may improve the accuracy of endometriosis diagnosis $(12,13,15,16,18)$, however no highly accurate marker for endometriosis has yet been detected. Therefore, the present study aimed to evaluate IL-6 levels in serum and PF, as well as IL-6 gene expression in subcutaneous and visceral fat, and in the eutopic and ectopic endometrium of patients with pelvic endometriosis. 


\section{Patients and methods}

Patients. Women who underwent gynecological laparoscopy in the Human Reproductive Unit of Hospital Fêmina (Porto Alegre, Brazil) between September 2007 and March 2009 and had a diagnosis of endometriosis confirmed during the procedure were enrolled in the current study. Endometriosis was diagnosed if atypical implants were observed during laparoscopy or confirmed by histological examination of sites exhibiting signs of active endometriosis. Women without evidence of pelvic disease according to laparoscopic and histopathological examinations were included in the control group (normal pelvis). Infertility was defined as absence of pregnancy after 1 year of sexual intercourse without contraception. Chronic pelvic pain was defined as non-cyclical pelvic pain occurring for $\geq 6$ months and severe enough to cause limitations to daily activities or require medical treatment. A total of 30 women with endometriosis and 18 women with a normal pelvis were included in the study. The inclusion criteria were: i) Women were pre-menopausal (age range, 21-50 years) and ii) had not received hormonal therapy for at least 3 months prior to surgery. Patients were excluded if they had a body mass index $(\mathrm{BMI})>35$. The study protocol was approved by the Research Ethics Committee at Hospital de Clínicas de Porto Alegre (Porto Alegre, Brazil) and written informed consent was obtained from all participants.

Study protocol. All the women in both groups underwent clinical examination. Laparoscopies were performed by the same surgeon, who diagnosed and classified endometriosis according to the revised American Society for Reproductive Medicine classification (r-ASRM) (19).

Gynecological laparoscopy was preferentially scheduled when the patients were at the secretory phase of the menstrual cycle, however a proportion of patientsunderwent the procedure during the proliferative phase (22.4\%). Dates of the menstrual cycle were confirmed by histological examination and defined according to the criteria described by Noyes et al (20). In 2 patients the endometrial biopsies were unsatisfactory and 1 patient underwent a hysterectomy prior to completion of the current study; therefore, these patients were excluded from the results and data analysis.

Peripheral venous blood samples were collected immediately prior to laparoscopy, and PF was collected immediately following the beginning of the procedure. Samples were stored at $-20^{\circ} \mathrm{C}$ until they were processed in the laboratory. Cell components from blood and PF were removed by centrifugation at $300 \mathrm{x}$ g for $20 \mathrm{~min}$. The supernatants were collected and stored in aliquots at $-80^{\circ} \mathrm{C}$ until assayed.

Serum hormone measurements. Serum estradiol and progesterone were assayed by electrochemiluminescence using an Elecsys 2010 system (Roche Diagnostics GmbH, Mannheim, Germany) according to the manufacturer's protocol. Intra and interassay coefficients of variation (CVs) were $>5 \%$.

Serum and PF IL-6 measurements. IL-6 levels were determined using the Human IL-6 Enzime Immunometric Assay Kit (Assay Designs, Inc., Ann Arbor, MI, USA) with a detection limit of $3.91 \mathrm{pg} / \mathrm{ml}$ and intra and interassay CVs of 5.3 and $12.4 \%$, respectively. Analyses were performed in duplicate in accordance with the manufacturer's instructions. Internal control samples from the kit were analysed for quality assurance.

Tissue collection. Eutopic and ectopic endometrial samples, as well as subcutaneous and visceral AT samples, were collected from the pelvic region before the end of the laparoscopy procedure: A single sample of superficial peritoneal endometriotic tissue was obtained from the largest lesion; subcutaneous and visceral AT samples $\left(\sim 1 \mathrm{~cm}^{3}\right)$ were harvested at the end of the procedure; and eutopic endometrial samples were harvested via curettage. Samples were immediately frozen in liquid nitrogen and stored at $-80^{\circ} \mathrm{C}$ until RNA isolation.

Reverse transcription-quantitative polymerase chain reaction (RT-qPCR). Total RNA was extracted from the samples using phenol/guanidine isothiocyanate (TRIzol; Invitrogen; Thermo Fisher Scientific, Inc., Waltham, MA, USA), as previously described (21). Concentration and quality of total RNA were assessed with a NanoDrop 2000 spectrophotometer (Thermo Fisher Scientific, Inc., Wilmington, DE, USA). Reverse transcription of $1 \mu \mathrm{g}$ total RNA into cDNA was performed using a SuperScript ${ }^{\circledR}$ II First-Strand Synthesis system for RT-PCR (Invitrogen; Thermo Fisher Scientific, Inc.) (21-23). qPCR was performed in triplicate, using the ViiA $^{\mathrm{TM}} 7$ Real-Time PCR system (Thermo Fisher Scientific, Inc.). qPCR protocol conditions consisted of denaturation at $95^{\circ} \mathrm{C}$ for $20 \mathrm{sec}$, followed by 50 cycles of $5 \mathrm{sec}$ at $95^{\circ} \mathrm{C}$ and $20 \mathrm{sec}$ at $60^{\circ} \mathrm{C}$. Experiments were performed by monitoring in real time the increase in fluorescence of the SYBR-Green dye (Applied Biosystems; Thermo Fisher Scientific, Inc.), as previously described (24-26). Primers were designed using Primer Express 3.0 Software for Real-Time PCR (Applied Biosystems; Thermo Fisher Scientific, Inc.) and acquired from Thermo Fisher Scientific, Inc. Forward and reverse primer sequences designed for IL-6 (NM_0,00600) were: Forward, 5'-CCACACAGACAGCCACTCA-3' and reverse, 5'-CATCCATCTTTTTCAGCCATCT-3'. The GAPDH gene (NM_001256799) was used to normalize mRNA expression. GAPDH primer sequences were: Forward, 5'-ACC CACTCCTCCACCTTTG-3' and reverse, 5'-CTCTTGTGC TCTTGCTGGG-3'. cDNA samples $(1.0 \mathrm{ng} / \mu \mathrm{l})$ were mixed with a predetermined forward and reverse primer volume (1.0 $\mu 1$ of each primer for IL-6, and $0.5 \mu 1$ of each primer for GAPDH) and 5.0 $\mu \mathrm{l}$ Fast SYBR-Green Master mix (Applied Biosystems; Thermo Fisher Scientific, Inc..) in a total reaction mixture of $10.0 \mu 1$. Primers generated amplicons that produced a single sharp peak during melting curve analysis. Data were analyzed by relative quantitation using the comparative $\Delta \Delta \mathrm{C}_{\mathrm{q}}$ method (27).

Statistical analysis. Results are presented as mean \pm standard deviation or median and interquartile range (25-75\%). Comparisons between means were analyzed using the unpaired Student's t-test or Mann-Whitney U test. Comparisons of median values involving three groups were analyzed using the Kruskal-Wallis test. The $\chi^{2}$ test was used to compare qualitative variables. Pearson's or Spearman's rank 
Table I. Distribution of patients with endometriosis and patients with a normal pelvis (control group) according to serum estradiol and progesterone levels and phase of the menstrual cycle.

\begin{tabular}{|c|c|c|c|c|}
\hline \multirow[b]{2}{*}{ Variable } & \multicolumn{2}{|c|}{ Endometriosis } & \multirow[b]{2}{*}{ Controls $(n=16)$} & \multirow[b]{2}{*}{ P-value } \\
\hline & Stage I/II $(n=14)$ & Stage III/IV $(n=16)$ & & \\
\hline \multicolumn{5}{|c|}{ Phase of cycle, n (\%) } \\
\hline Proliferative & $4(28.6)$ & $3(18.8)$ & $4(22.2)$ & \\
\hline Secretory & $10(71.4)$ & $13(81.2)$ & $12(66.7)$ & \\
\hline \multicolumn{5}{|c|}{ Estradiol $(\mathrm{pg} / \mathrm{ml})^{\mathrm{b}}$} \\
\hline Proliferative & $122(11-164)$ & $157(63-169)$ & $65(18-144)$ & 0.55 \\
\hline Secretory & $143(65-231)$ & $102(42-214)$ & $91(55-132)$ & 0.64 \\
\hline \multicolumn{5}{|c|}{ Progesterone $(\mathrm{ng} / \mathrm{ml})^{\mathrm{b}}$} \\
\hline Proliferative & $0.20(0.16-0.79)$ & $0.73(0.20-0.80)$ & $0.42(0.21-0.86)$ & 0.71 \\
\hline Secretory & $6.12(0.92-13.22)$ & $1.05(0.37-9.50)$ & $4.15(0.59-9.16)$ & 0.44 \\
\hline
\end{tabular}

${ }^{a}$ Kruskall-Wallis; ${ }^{b}$ Data presented as median (interquartile range).

correlation coefficients were calculated between variables using a two-tailed test for significance. $\log _{10}$ transformation was used to normalize the distribution of non-Gaussian variables and mean values were back-transformed for presentation. $\mathrm{P}<0.05$ was considered to indicate a statically significant difference. The Statistical Package for the Social Sciences version 18 (SPSS, Inc., Chicago, IL, USA) was used to perform analyses.

\section{Results}

Clinical and hormonal profile of participants. Patients with endometriosis and women with a normal pelvis had a similar age $(32.6 \pm 7.4$ vs. $33 \pm 5.8$ years, $\mathrm{P}=0.98)$ and $\mathrm{BMI}(25.5 \pm 4.5$ vs. $\left.25.2 \pm 3.5 \mathrm{~kg} / \mathrm{m}^{2}, \mathrm{P}=0.86\right)$. Table I presents the serum estradiol, progesterone levels and phase of the menstrual cycle for patients with endometriosis and women with a normal pelvis, which were similar in the two groups $(\mathrm{P}>0.05)$.

Endometriosis was classified as minimal (stage I) in 14 patients, moderate (stage III) in 7 patients and severe (stage IV) in 10 patients (Table I). Active endometriosis, characterized by red lesions, was predominant in $16(53.3 \%)$ patients; black and white lesions were observed in $10(33.3 \%)$ patients and endometrioma as the primary observation in laparoscopy was detected in $4(13.3 \%)$ patients. In the endometriosis group, laparoscopy was performed for infertility in 11 patients $(36.7 \%)$, pelvic pain in 10 patients $(33.3 \%)$, adnexal pathology in 7 patients $(23.3 \%)$ and tubal ligation in 2 patients $(6.6 \%)$. In the control group, laparoscopy was performed for tubal ligation in 13 patients $(72.2 \%)$, infertility in 4 patients $(22.2 \%)$ and for both tubal ligation and pelvic pain in 1 patient $(5.6 \%)$.

IL-6 levels in serum and PF. IL-6 levels were detected in the PF of all patients for whom this cytokine was measured (25 patients with endometriosis and 16 control women). IL-6 levels in the PF were significantly higher in the endometriosis group compared with the controls $(\mathrm{P}=0.047$;
Fig. 1A). In addition, IL-6 concentrations in the PF were significantly higher in patients with endometriosis stage III/ IV than in patients with endometriosis stage I/II and in the control group $(\mathrm{P}<0.001$; Fig. 1B). However, there were no significant differences in PF IL-6 levels between patients with endometriosis of stage I/II and the control group. Fig. 2 demonstrates a strong correlation between IL-6 levels in PF and the r-ASRM (19) scores of endometriosis severity $(\mathrm{r}=0.77 ; \mathrm{P}=0.001)$. Regarding IL- 6 serum levels, the majority of the samples exhibited results below the limit of detection of the assay. Only 11 patients, eight in the endometriosis group (mean, $10.2 \mathrm{pg} / \mathrm{ml}$; range, $8.5-12.6 \mathrm{pg} / \mathrm{ml}$ ) and three in the control group (mean, $9.5 \mathrm{pg} / \mathrm{ml}$; range, $9-12.4 \mathrm{pg} / \mathrm{ml}$ ) $(\mathrm{P}=0.77)$ exhibited IL-6 concentrations $>3.10 \mathrm{pg} / \mathrm{ml}$.

IL-6 gene expression in eutopic and ectopic endometrium and in subcutaneous and visceral AT. Expression of IL-6 mRNA did not differ significantly between patients and controls in subcutaneous or visceral AT (Fig. 3A). The mRNA expression of IL-6 was similar in the eutopic and ectopic endometrial tissue of patients with endometriosis, as well as in the endometrium of women with normal pelvis (Fig. 3B).

\section{Discussion}

In the present study, IL-6 levels in the PF of patients with endometriosis were significantly increased compared with those in women with a normal pelvis. In addition, patients with moderate and severe forms of the disease had significantly higher levels of IL-6 in the PF than were observed in patients with mild endometriosis and controls. Furthermore, a strong positive correlation was observed between IL-6 levels in the PF and endometriosis severity.

Current available data on literature are inconsistent regarding the association between IL-6 and pelvic endometriosis. Indeed, the results of the present study partially agree with the findings reported by Velasco et al (9), which 
A

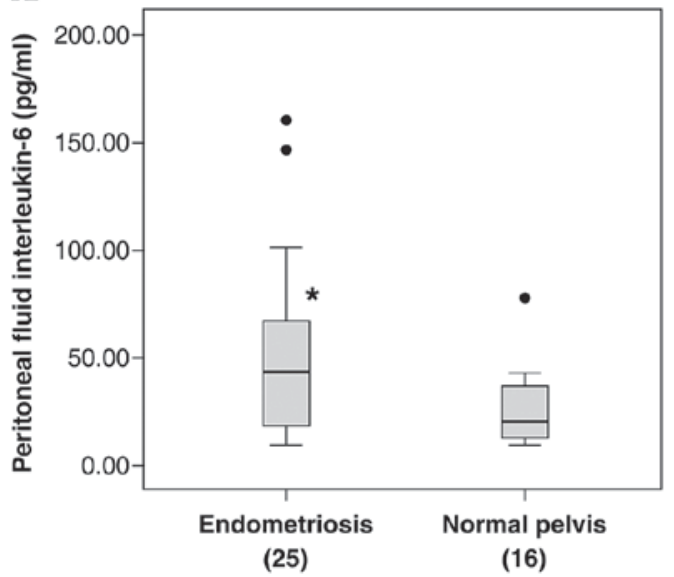

B

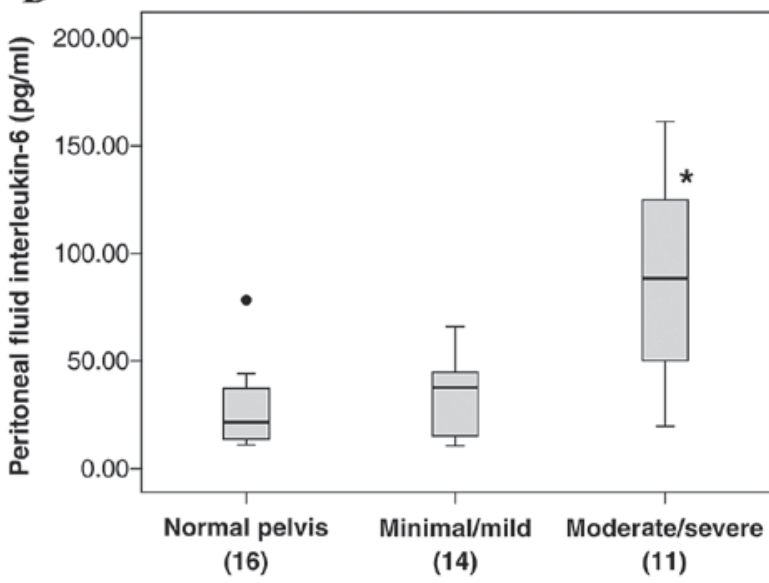

Figure 1. Peritoneal fluid interleukin-6 concentrations (A) in patients with endometriosis $(\mathrm{n}=25)$ and women with normal pelvis (control group; $\mathrm{n}=16)$, $\mathrm{P}=0.047$ (Mann-Whitney U test) and (B) stratified according to the stage of endometriosis according to the revised American Society for Reproductive Medicine classification (19), ${ }^{*} \mathrm{P}<0.001$ vs. minimal/mild and normal pelvis groups (Mann-Whitney U test). ${ }^{\circ}$ Outlier values.

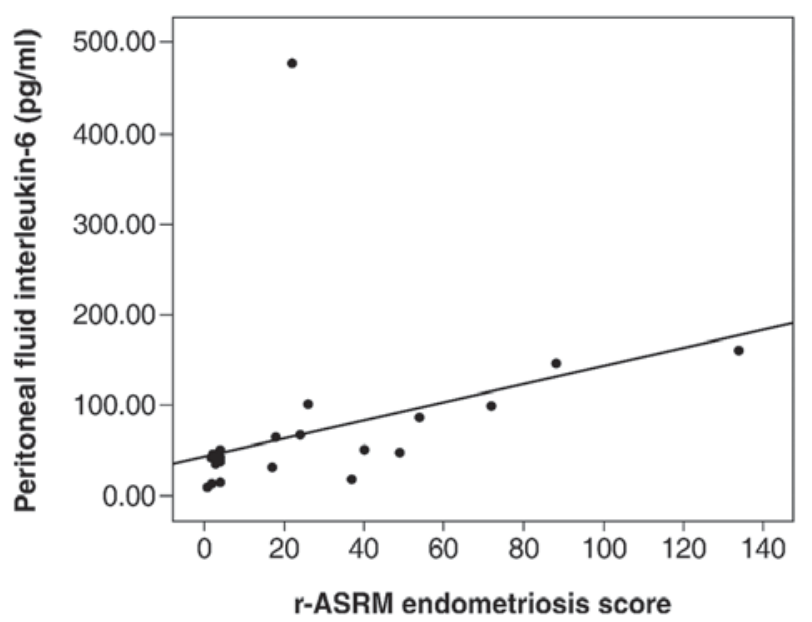

Figure 2. Association between peritoneal fluid interleukin-6 and r-ASRM classification scores (19) in patients with endometriosis. A positive correlation was detected $(r=0.77, \mathrm{P}<0.001)$. r-ASRM, revised American Society for Reproductive Medicine.

described increased IL-6 levels in the PF of patients with moderate or severe endometriosis but did not identify a correlation between IL-6 and endometriosis severity. This may be explained by the fact that only patients with more advanced stages of endometriosis were included in this previous study. Punnonen et al (7) reported increased levels of IL-6 and IL-10 in the PF of patients with endometriosis and also did not identify any correlation between IL-6 levels and disease severity. Furthermore, Khan et al (10) did not observe any differences in IL-6 levels in the PF between patients with endometriosis and controls without the disease. Laparoscopy demonstrated that patients with red lesions had higher levels of hepatocyte growth factor (HGF), IL-6, progesterone and estradiol than patients with black and white lesions or controls (10). It is widely considered that atypical lesions (red) are more biologically active than typical ones (black) (28). Patients in the early stages of endometriosis with more active lesions may have an increased level of cytokine secretion that may explain the symptoms of pain and infertility. Indeed, the level of endometriosis lesion activity, rather than the lesion extension appears to be a more accurate parameter of disease severity. In a previous study, Harada et al (8) demonstrated that PF levels of IL-6, IL-6 soluble receptor and the tumor necrosis factor- $\alpha(\mathrm{TNF}-\alpha)$ were correlated with the number and size of endometrial implants in infertile patients. In addition to increased levels of IL-6 and TNF- $\alpha$ in patients with endometriosis, a positive correlation between the number of red lesions and cytokine levels was observed (8). It was therefore suggested that increased levels of cytokines in PF may be associated with infertility and the pathogenesis of endometriosis and that the production of IL- 6 may be the result of stimulation by increased levels of TNF- $\alpha$ (8). These results are in accordance with the results of the present study, which demonstrated that endometriosis samples were characterized by active peritoneal and ovarian lesions in $70 \%$ of patients. This may be, at least in part, the reason why a significant association between PF IL-6 levels and the severity of endometriosis was detected.

One study reported levels of IL-6 in PF obtained in the secretory phase of the cycle were higher than those in the proliferative phase (8); however, other studies have not found significant differences in PF or serum cytokine levels between proliferative or secretory phases $(10,17)$. In the present study, laparoscopies were preferentially scheduled in the secretory phase to assess the association between IL-6 levels and endometrial classification, rather than proliferation. However, the material from some participants was collected in the proliferative phase, and serum and PF IL-6 levels could be compared in the two phases for patients with endometriosis and control participants. The results of the current study suggest that IL- 6 production is not modulated by steroid hormone fluctuations during the menstrual cycle.

Different cut-off values for serum levels of IL-6 are indicated to be good predictors of endometriosis $(14,17,29)$. Others have suggested that an association of serum markers may increase diagnostic accuracy $(13,18)$. The observations recorded in the current study are in accordance with those reported by Kalu et al (30), which did not detect any differences in the serum IL-6 levels of patients with endometriosis 
A

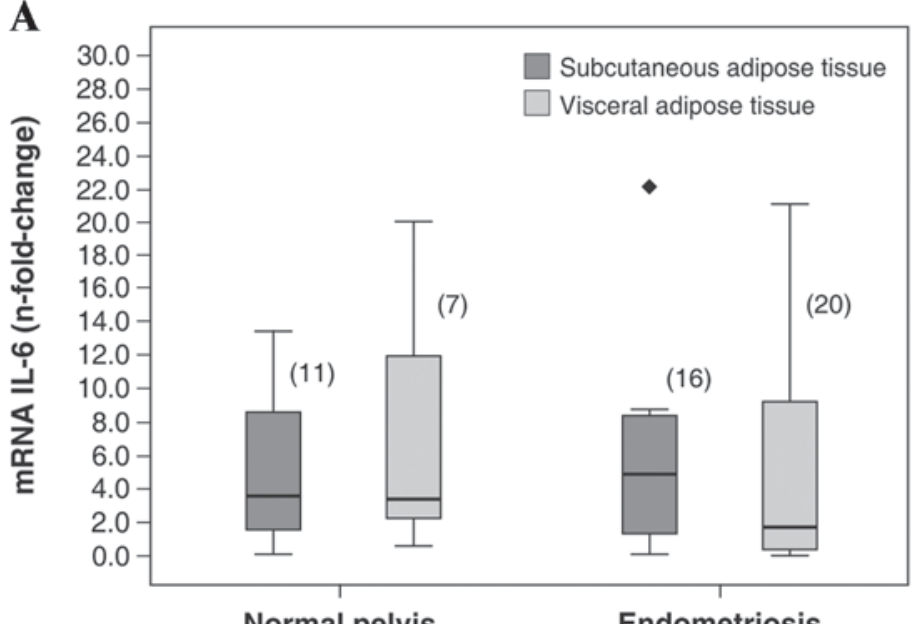

B

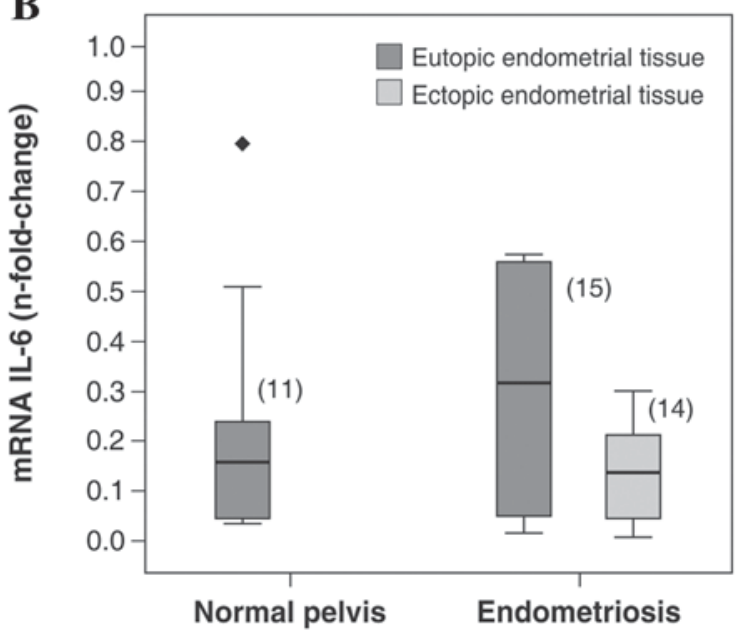

Figure 3. IL-6 gene expression (A) in subcutaneous and visceral adipose tissue and (B) in endometrial tissue, of patients with endometriosis and normal pelvis (control group). Levels of mRNA were expressed as n-fold-change differences from the calibrator sample $\left(\Delta \Delta \mathrm{C}_{\mathrm{q}}\right.$ method). The number of biopsies analyzed per group appears within parentheses. Values are expressed as median and interquartile range (25-75\%). 'Outlier values. IL-6, interleukin-6.

in comparison with patients without endometriosis. One limitation of the present study was that levels of IL- 6 were detectable in the samples of some but not all patients due to the test detection limit. In addition, variations in assay methods and/or patient selection may account for these differences.

In the present study, the correlation between levels of IL-6 and the gene expression of IL- 6 in endometrial tissue was investigated. No differences were found in IL-6 gene expression either in the ectopic or eutopic endometrium of patients with endometriosis compared with women with a normal pelvis. It was previously demonstrated that the expression of IL-6 on endometrium may be increased in ectopic tissue (31), and Tsudo et al (32) detected a significant increase in IL-6 expression in endometriotic tissue derived from stromal cells. These differences in results from different studies may be due, at least in part, to the types of lesions being assessed in each study, which included peritoneal, ovarian or deeply infiltrating endometriosis lesions. Furthermore, results from different studies indicate that different lesions may be considered as separate entities with different pathogenesis $(32,33)$. Therefore, further studies are required to additionally assess IL-6 protein expression on endometrium, as an altered post-transcriptional control system may be operative in these circumstances.

In the current study, no differences in IL-6 gene expression were detected either in the visceral or subcutaneus AT of patients with endometriosis compared with women with a normal pelvis. This means that the source of higher IL-6 levels in the PF of patients with endometriosis may not to be associated with regional AT. Other cells, including peritoneal macrophages and leukocites may be associated with the local PF production of IL-6 as previously reported (32), which merits further investigation.

In conclusion, the present results suggest that levels of IL-6 may be associated with the presence and severity of endometriosis. Further studies should evaluate IL-6 protein expression in ectopic and eutopic endometrium in order to elucidate the role of this cytokine in the pathogenesis of endometriosis.

\section{Acknowledgements}

This study was supported by grants from the National Council for Scientific and Technological Development (CNPq INCT 573747/2008-3) and the Fund for Research Support, Hospital de Clínicas de Porto Alegre (FIPE-HCPA 07-339), Brazil.

\section{References}

1. Burney RO and Giudice LC: Pathogenesis and pathophysiology of endometriosis. Fertil Steril 98: 511-519, 2012.

2. Trayhurn P and Wood IS: Adipokines: Inflammation and the pleiotropic role of white adipose tissue. Br J Nutr 92: 347-355, 2004.

3. Proença AR, Sertié RA, Oliveira AC, Campaña AB, Caminhotto RO, Chimin P and Lima FB: New concepts in white adipose tissue physiology. Braz J Med Biol Res 47: 192-205, 2014

4. Motro B, Itin A, Sachs L and Keshet E: Pattern of interleukin 6 gene expression in vivo suggests a role for this cytokine in angiogenesis. Proc Natl Acad Sci USA 87: 3092-3096, 1990.

5. Nothnick WB: Treating endometriosis as an autoimmune disease. Fertil Steril 76: 223-231, 2001.

6. Akoum A, Lemay A, Paradis I, Rheault N and Maheux R: Secretion of interleukin- 6 by human endometriotic cells and regulation by proinflammatory cytokines and sex steroids. Hum Reprod 11: 2269-2275, 1996.

7. Punnonen J, Teisala K, Ranta H, Bennett B and Punnonen R: Increased levels of interleukin- 6 and interleukin-10 in the peritoneal fluid of patients with endometriosis. Am J Obstet Gynecol 174: 1522-1526, 1996.

8. Harada T, Yoshioka H, Yoshida S, Iwabe T, Onohara Y, Tanikawa $\mathrm{M}$ and Terakawa N: Increased interleukin-6 levels in peritoneal fluid of infertile patients with active endometriosis. Am J Obstet Gynecol 176: 593-597, 1997.

9. Velasco I, Acién P, Campos A, Acién MI and Ruiz-Maciá E: Interleukin-6 and other soluble factors in peritoneal fluid and endometriomas and their relation to pain and aromatase expression. J Reprod Immunol 84: 199-205, 2010.

10. Khan KN, Masuzaki H, Fujishita A, Hamasaki T, Kitajima M, Hasuo A, Miyamura Y and Ishimaru T: Association of interleukin-6 and estradiol with hepatocyte growth factor in peritoneal fluid of women with endometriosis. Acta Obstet Gynecol Scand 81: 764-771, 2002. 
11. Bedaiwy MA, El-Nashar SA, Sharma RK and Falcone T: Effect of ovarian involvement on peritoneal fluid cytokine concentrations in endometriosis patients. Reprod Biomed Online 14: 620-625, 2007.

12. Gagne D, Rivard M, Pagé M, Lépine M, Platon C, Shazand K, Hugo P and Gosselin D: Development of a nonsurgical diagnostic tool for endometriosis based on the detection of endometrial leukocyte subsets and serum CA-125 levels. Fertil Steril 80: 876-885, 2003

13. Somigliana E, Viganò P, Tirelli AS, Felicetta I, Torresani E, Vignali $\mathrm{M}$ and Di Blasio AM: Use of the concomitant serum dosage of CA 125, CA 19-9 and interleukin-6 to detect the presence of endometriosis. Results from a series of reproductive age women undergoing laparoscopic surgery for benign gynaecological conditions. Hum Reprod 19: 1871-1876, 2004.

14. Martinez S, Garrido N, Coperias JL, Pardo F, Desco J, García-Velasco JA, Simón C and Pellicer A: Serum interleukin-6 levels are elevated in women with minimal-mild endometriosis. Hum Reprod 22: 836-842, 2007.

15. Agic A, Djalali S, Wolfler MM, Halis G, Diedrich K and Hornung D: Combination of CCR1 mRNA, MCP1, and CA125 measurements in peripheral blood as a diagnostic test for endometriosis. Reprod Sci 15: 906-911, 2008.

16. Seeber B, Sammel MD, Fan X, Gerton GL, Shaunik A, Chittams $J$ and Barnhart KT: Panel of markers can accurately predict endometriosis in a subset of patients. Fertil sterilit 89: 1073-1081, 2008.

17. Othman Eel D, Hornung D, Salem HT, Khalifa EA, El-Metwally TH and Al-Hendy A: Serum cytokines as biomarkers for nonsurgical prediction of endometriosis. Eur J Obstet Gynecol Reprod Biol 137: 240-246, 2008.

18. Mihalyi A, Gevaert O, Kyama CM, Simsa P, Pochet N, De Smet F, De Moor B, Meuleman C, Billen J, Blanckaert N, et al: Non-invasive diagnosis of endometriosis based on a combined analysis of six plasma biomarkers. Hum Reprod 25: 654-664, 2010.

19. Revised American Society for Reproductive Medicine classification of endometriosis: 1996. Fertil Steril 67: 817-821, 1997.

20. Noyes R, Heting A and Rock J: Dating the endometrial biopsy. Fertil Steril 1: 3-25, 1950.

21. Nácul AP, Lecke SB, Edelweiss MI, Morsch DM and Spritzer PM: Gene expression of leptin and long leptin receptor isoform in endometriosis: A case-control study. Obstet Gynecol Int 2013: 879618, 2013

22. Lecke SB, Mattei F, Morsch DM and Spritzer PM: Abdominal subcutaneous fat gene expression and circulating levels of leptin and adiponectin in polycystic ovary syndrome. Fertil Steril 95 2044-2049, 2011.
23. Lecke SB, Morsch D and Spritzer PM: Circulating levels and subcutaneous adipose tissue gene expression of pigment epithelium-derived factor in polycystic ovary syndrome and normal women: A case control study. Reprod Biol Endocrinol 11: 77, 2013.

24. Higuchi R, Dollinger G, Walsh PS and Griffith R: Simultaneous amplification and detection of specific DNA sequences. Biotechnology (N Y) 10: 413-417, 1992.

25. Higuchi R, Fockler C, Dollinger G and Watson R: Kinetic PCR analysis: Real-time monitoring of DNA amplification reactions. Biotechnology (N Y) 11: 1026-1030, 1993.

26. Zipper H, Brunner H, Bernhagen J and Vitzthum $F$ : Investigations on DNA intercalation and surface binding by SYBR Green I, its structure determination and methodological implications. Nucleic Acids Res 32: e103, 2004.

27. Livak KJ and Schmittgen TD: Analysis of relative gene expression data using real-time quantitative PCR and the 2(-Delta Delta C(T)) Method. Methods 25: 402-408, 2001

28. Khan KN, Masuzaki H, Fujishita A, Kitajima M, Sekine I and Ishimaru T: Higher activity by opaque endometriotic lesions than nonopaque lesions. Acta Obstet Gynecol Scand 83: 375-382, 2004

29. Bedaiwy MA, Falcone T, Sharma RK, Goldberg JM, Attaran M, Nelson DR and Agarwal A: Prediction of endometriosis with serum and peritoneal fluid markers: A prospective controlled trial. Hum Reprod 17: 426-431, 2002.

30. Kalu E, Sumar N, Giannopoulos T, Patel P, Croucher C, Sherriff E and Bansal A: Cytokine profiles in serum and peritoneal fluid from infertile women with and without endometriosis. J Obstet Gynaecol Res 33: 490-495, 2007.

31. Salmassi A, Acil Y, Schmutzler AG, Koch K, Jonat W and Mettler L: Differential interleukin-6 messenger ribonucleic acid expression and its distribution pattern in eutopic and ectopic endometrium. Fertil Steril 89 (5 Suppl): S1578-S1584, 2008

32. Tsudo T, Harada T, Iwabe T, Tanikawa M, Nagano Y, Ito M Taniguchi $\mathrm{F}$ and Terakawa $\mathrm{N}$ : Altered gene expression and secretion of interleukin- 6 in stromal cells derived from endometriotic tissues. Fertil Steril 73: 205-211, 2000.

33. Nisolle M and Donnez J: Peritoneal endometriosis, ovarian endometriosis, and adenomyotic nodules of the rectovaginal septum are three different entities. Fertil Steril 68: 585-596, 1997. 